\title{
Omnidirectional Analysis of Spatial Manipulator
}

\author{
Yuquan Leng, ${ }^{1,2}$ Yang Zhang, ${ }^{1}$ Xu He, ${ }^{1}$ Wei Zhang, ${ }^{1}$ Haitao Luo, ${ }^{1}$ and Weijia Zhou ${ }^{1}$ \\ ${ }^{1}$ State Key Laboratory of Robotics, Shenyang Institute of Automation, Shenyang 110016, China \\ ${ }^{2}$ University of Chinese Academy of Science, Beijing, China \\ Correspondence should be addressed to Yuquan Leng; lengyuquan@sia.cn
}

Received 12 November 2014; Accepted 15 April 2015

Academic Editor: Shahram Payandeh

Copyright (C) 2015 Yuquan Leng et al. This is an open access article distributed under the Creative Commons Attribution License, which permits unrestricted use, distribution, and reproduction in any medium, provided the original work is properly cited.

\begin{abstract}
Space manipulators are mainly used in the spatial loading task. According to problems of the spatial loading diversity, the testing loading installing position, and the utilization ratio of a test platform, the space manipulator is asked to evaluate the position and attitude of itself. This paper proposes the Point Omnidirectional Coefficient (POC) with unit attitude sphere/circle to describe attitude of the end-effector, which evaluates any points in the attainable space of the manipulators, in combination with the manipulation's position message, and get relationships between its position and attitude of all points in the attainable space. It represents the mapping between sphere surface and plane for mission attitude constraints and the method for calculating volume of points space including attainable space, Omnidirectional space, and mission attitude space. Furthermore, the Manipulator Omnidirectional Coefficient based on mission or not is proposed for evaluating manipulator performance. Through analysis and simulation about 3D and 2D manipulators, the results show that the above theoretical approach is feasible and the relationships about link lengths, joints angles, attainable space, and Manipulator Omnidirectional Coefficient are drawn for guiding design.
\end{abstract}

\section{Introduction}

Manipulators research on its Omnidirectional characteristics is important and meaningful for manipulators used in space [1-4]. Space manipulators can take place of spacemen to complete many assignments. As its operational environment is special, there is urgent need for Omnidirectional analysis. Spatial loading has the following problems: first, the operation method differs from one loading to another, and it also differs for operational position and pose; second, the problem is how to install the loading appropriately in order to complete the task; last, according to the need of the loading task, the problem is how to distribute the loading properly in the test platform to maximize utilization of the platform. The attainable space of manipulator refers to all points that the manipulator end-effector can reach with all joints in their proper domain $[5,6]$.

In this paper, the POC means the proportion of attitude manipulator can arrive at this point. The bigger the POC is, the bigger the attitude can reach in one point. The POC being 1 means that attitude is attainable Omnidirectionally. Omnidirectional space of the manipulator means the unit of points where the POC is 1 , where the manipulator is the most flexible. The research about Omnidirectional characteristics analyzes position data of its reachable workspace combined with attitude data and could make an accurate evaluation of its performance itself. The difference between attainable space and the Omnidirectional space represents the space where the position is attainable and attitude of manipulator is attainable partly.

Specific mission operation does not require all attitudes. We define mission attitude space, which means the space meets mission attitude operation. It is obvious that mission attitude space includes Omnidirectional space. This paper proposes mapping relationship from spherical surface to plane, realizing mission attitude constraints.

We calculate volume of workspace, mission attitude space, and Omnidirectional space with numerical analysis and analytical method $[5,7,8]$. Numerical analysis is universal and does not become complicated with complexity of the manipulator. Analytical method is complicated and not universal related with nonlinear equations and inverse matrix of manipulator kinematics $[6,9,10]$. This paper applies numerical analysis to calculate space consisting of points, 


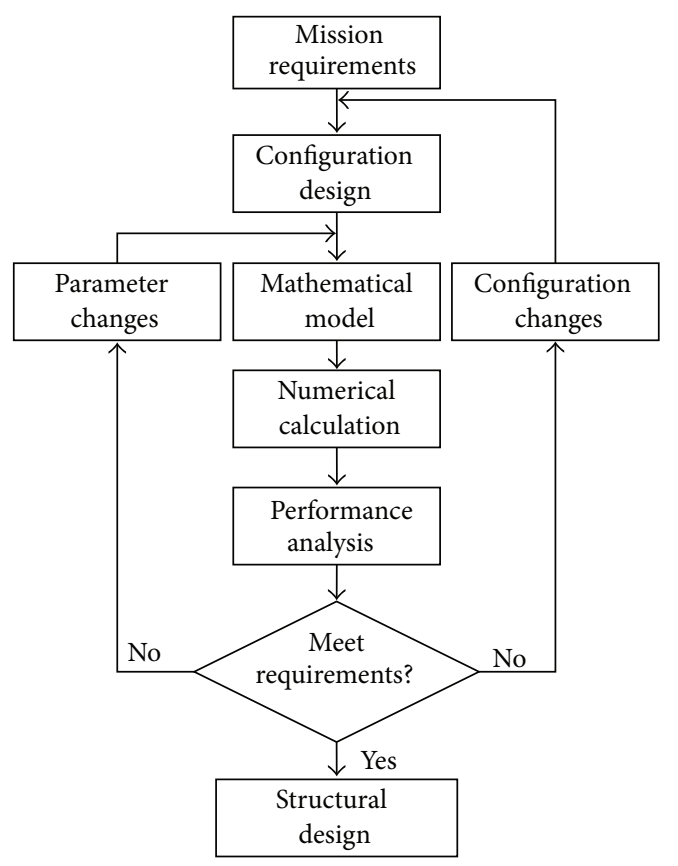

Figure 1: Design process based on mission requirements.

which is generated using the Monte Carlo method [11, 12]. The two-dimensional point's distributions of each layer of the space are obtained by classifying these points into a series of slices. Point's areas are achieved by using rectangle method, and then volume of point's space is achieved.

Combined with the loading task, the research about attainable space, mission attitude space, and Omnidirectional space, on one hand, as shown in Figure 1, can solve many problems in loading installation and avoid the task being unfinished when the loading has been installed; furthermore, it can also arrange distribution of loading on the test platform [13]. On the other hand, as shown in Figure 2, according to given assignment, it can be used to represent flexibility of the whole manipulator. When the manipulator meets task requirements, it will perform task; otherwise it will give feedback to human about fail result and suggestions. Consequently, it has guiding significance to joint angles, link lengths, and other parameters of the manipulator [14-17].

In Section 2 of this paper, the early researches of manipulator performance are introduced, which makes an equivalent representation about the Omnidirectional characteristics; Section 3 introduced the method to analyze the Omnidirectional characteristics, including the POC analysis, the mission attitude constraints, the volume of point's space, and the Manipulator Omnidirectional Coefficient; Section 4 shows the result of data analysis and figures of 3D manipulator and 2D manipulator.

\section{Prior Work}

Salisbury and Craig proposed condition number for the dexterity of manipulators. The Singular Value Decomposition (SVD) method works for Jacobian matrix of all kinematic

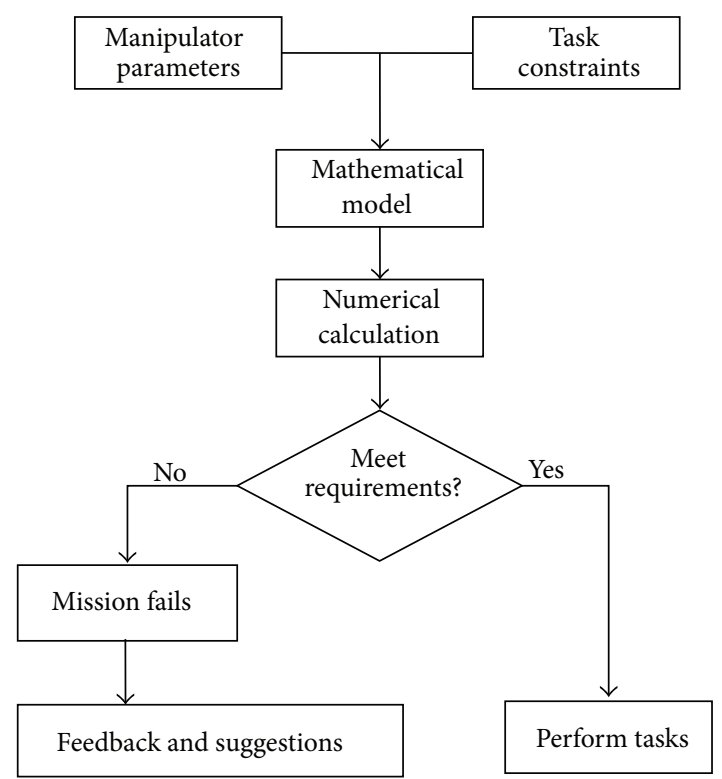

Figure 2: Analysis about whether the mission can be completed based on a specific mission and manipulator.

structures; then condition number can be achieved [18]. Yoshikawa gave one way to describe the manipulability of robotic arm in positioning and orienting end-effector. $\mathrm{He}$ introduced the term manipulability which involves the Jacobian matrix and its transpose. The manipulability measure is determined by the evaluation of the determinant of the Jacobian [8]. Klein and Blaho introduced four measures for the dexterity of manipulators: determinant, condition number, and minimum singular value of Jacobian and joints range availability. They compared four measures to achieve the advantage and disadvantage of each for finding an optimal configuration [19]. Mohammed et al. studied the manipulability index for every point using a new algorithm based on the way of condition number [6].

Van Den Doel and Pai showed a performance measure of robot manipulators based on differential geometry. Two- and three-link planar arms are analyzed with this measure [20]. Guo and Gao proposed graphical method to display global performances of manipulators for planar arm [21,22].

All of the above methods can describe one or more performances of the robot arm which include dexterous, manipulability, and sensitivity and are inferior for representing Omnidirectional characteristics. In addition, the calculation becomes more difficult with complexity of the manipulator.

\section{Omnidirectional Characteristics Analysis}

The reasonable distribution of loading on spatial test platform is based on exact Omnidirectional information about the manipulator. The early analytic methods reflected Omnidirectional information to some extent and could be used in guiding manipulators designing but not in actual controlling. The Omnidirectional Coefficient method mentioned in this paper is able to provide the information of both the attainable 
space and the corresponding point attitude and then provide basis for loading operation. We could build a database for system about Omnidirectional information, which will be accessed when the manipulator executes someone task.

3.1. POC of $3 D$ Manipulator. We put forward the POC and attitude sphere surface with unit radius to explain the coefficient as shown in Figure 3. We can use surface to describe any attitude of an end-effector in one point. POC is proportion reached of total attitude. If Omnidirectional Coefficient is 1 , then the unit of the Omnidirectional point is Omnidirectional space.

The position and attitude of end-effector can be expressed relative to the world coordinate system as (1) for $n \mathrm{DOF}$ manipulator. We all know that position and attitude are only depending on joint angle parameters $\theta_{i}(i=1, \ldots, n)$, on the condition that robot structural parameters are determined, so we could achieve all Omnidirection information:

$$
\begin{aligned}
& { }_{i}^{0} T=\left[\begin{array}{cccc}
f_{11}\left(\theta_{i}\right) & f_{12}\left(\theta_{i}\right) & f_{13}\left(\theta_{i}\right) & X\left(\theta_{i}\right) \\
f_{21}\left(\theta_{i}\right) & f_{22}\left(\theta_{i}\right) & f_{23}\left(\theta_{i}\right) & Y\left(\theta_{i}\right) \\
f_{31}\left(\theta_{i}\right) & f_{32}\left(\theta_{i}\right) & f_{33}\left(\theta_{i}\right) & Z\left(\theta_{i}\right) \\
0 & 0 & 0 & 1
\end{array}\right], \\
& (i=1, \ldots, n) .
\end{aligned}
$$

Posture is one-to-one attitude, so when we calculate attitude, we have known position. We can express it as (2). In the equation, $a, b$, and $c$ are constants and mean position of point, and $\mathbf{R}_{3 \times 3}$ is an attitude matrix, which can describe any attitude of end-effector:

$$
\begin{aligned}
\mathbf{R} & =\left[\begin{array}{lll}
f_{11}\left(\theta_{i}\right) & f_{12}\left(\theta_{i}\right) & f_{13}\left(\theta_{i}\right) \\
f_{21}\left(\theta_{i}\right) & f_{22}\left(\theta_{i}\right) & f_{23}\left(\theta_{i}\right) \\
f_{31}\left(\theta_{i}\right) & f_{32}\left(\theta_{i}\right) & f_{33}\left(\theta_{i}\right)
\end{array}\right], \\
X\left(\theta_{i}\right) & =a \\
Y\left(\theta_{i}\right) & =b \\
Z\left(\theta_{i}\right) & =c .
\end{aligned}
$$

The terminal coordinate system is defined on the endeffector. We draw the unit attitude sphere surface with the origin of terminal coordinate system as the center as shown in Figure 3. The coordinate system $X-Y-Z$ means world coordinate system. The coordinate system $X_{i}-Y_{i}-Z_{i}$ means terminal coordinate system. We define $X_{i}-Y_{i}-Z_{i}$ as attitude of end-effector, which can be achieved through rotation. In Figure 3, $\alpha$ means the rotational angle around the $X$ axis; $\beta$ means the rotational angle around the $Y$ axis; and $\gamma$ means the rotational angle around the $Z$ axis.

Figure 3 shows that the end-effector can get to any attitude by rotating. We can get the relation between attitude and $\alpha, \beta, \gamma$ as follows.

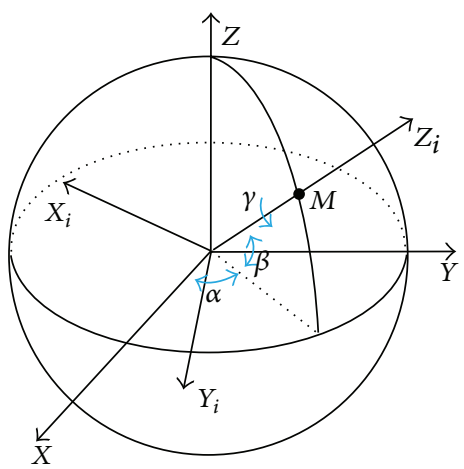

FIGURE 3: Attitude sphere surface.

When

$$
\begin{aligned}
& \sqrt{f_{31}\left(\theta_{i}\right)^{2}+f_{32}\left(\theta_{i}\right)^{2}} \neq 0, \\
& \sqrt{f_{31}\left(\theta_{i}\right)^{2}+f_{32}\left(\theta_{i}\right)^{2}} \neq 0,
\end{aligned}
$$

then

$$
\begin{aligned}
& \beta=A \tan 2\left(\sqrt{f_{31}\left(\theta_{i}\right)^{2}+f_{32}\left(\theta_{i}\right)^{2}}, f_{33}\left(\theta_{i}\right)\right), \\
& \alpha=A \tan 2\left(f_{23}\left(\theta_{i}\right), f_{13}\left(\theta_{i}\right)\right), \\
& \gamma=A \tan 2\left(f_{32}\left(\theta_{i}\right),-f_{31}\left(\theta_{i}\right)\right) ;
\end{aligned}
$$

when

$$
\begin{aligned}
\sqrt{f_{31}\left(\theta_{i}\right)^{2}+f_{32}\left(\theta_{i}\right)^{2}} & =0, \\
f_{33}\left(\theta_{i}\right) & >0,
\end{aligned}
$$

then

$$
\begin{aligned}
& \beta=0, \\
& \alpha=0, \\
& \gamma=A \tan 2\left(-f_{21}\left(\theta_{i}\right), f_{11}\left(\theta_{i}\right)\right) ;
\end{aligned}
$$

when

$$
\begin{array}{r}
\sqrt{f_{31}\left(\theta_{i}\right)^{2}+f_{32}\left(\theta_{i}\right)^{2}}=0 \\
f_{33}\left(\theta_{i}\right)<0
\end{array}
$$

then

$$
\begin{aligned}
& \beta=0, \\
& \alpha=0, \\
& \gamma=A \tan 2\left(f_{21}\left(\theta_{i}\right),-f_{11}\left(\theta_{i}\right)\right) .
\end{aligned}
$$

Attitude can also be indicated by the intersection $M$ and angle $\gamma$, where $M$ is the intersection of $Z_{i}$ axis and attitude sphere surface. $\gamma$ depends on the rotational extent of the $n$ joint, which has been assumed as $[-\pi \pi]$ in this paper. 


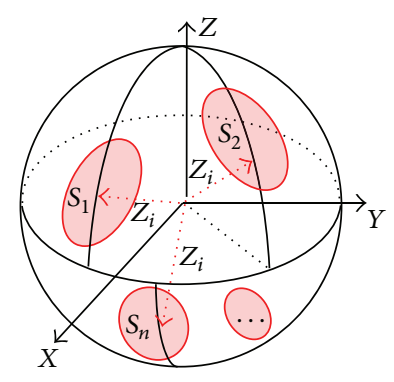

FIGURE 4: Reached areas to the spherical area.

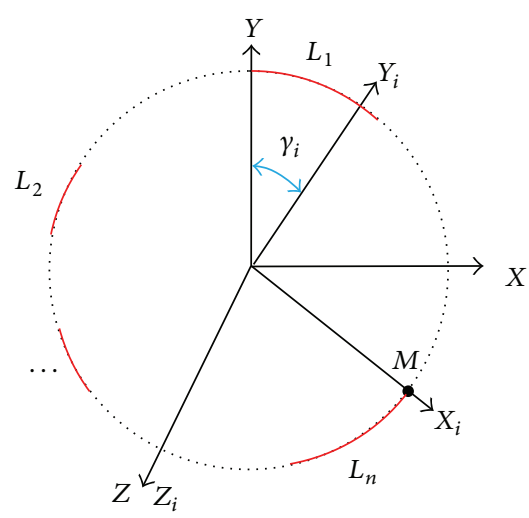

Figure 5: Attitude circle.

As attitude is changing, a unit of points has taken shape. POC can be represented as the proportion of the unit area to the spherical area, as shown in Figure 4, and expressed as

$$
D=\frac{\sum_{m=1}^{n} S_{m}(\alpha, \beta)}{S} .
$$

There may be more than one attainable domain for attitude on attitude sphere. $n$ means the quantity of domain; $S_{m}(\alpha, \beta)$ means the area of the $m$ th attainable domain. $S$ means the area of the whole attitude sphere. $D=1$ means that, at this point, any attitude can be reached with the joints in their rotational extent. The unit of point that can make $D=1$ is called Omnidirectional space.

3.2. POC of $2 \mathrm{D}$ Manipulator. $2 \mathrm{D}$ manipulator is the special form of 3D manipulator. In the analysis of 2D manipulator, we use planar circle instead of sphere surface. The circle in which the center is located in the end coordinate and the radius is
1 and is located on the manipulator surface is called attitude circle. As shown in Figure 5, $X-Y-Z$ coordinate system means attitude of world coordinate; $X_{i}-Y_{i}-Z_{i}$ coordinate system means attitude of end-effector. $\gamma$ means the rotational angle around the $Z$ axis. Attitude of end-effector can be represented by the intersection $M$ of the $X_{i}$ axis and attitude circle. The Point Omnidirectional Coefficient is the proportion of arc length of intersection unit to the circle length and can be shown in

$$
D=\frac{\sum_{m=1}^{n} L_{m}(\gamma)}{L}
$$

There may be more than one attainable line for attitude on attitude circle. $n$ means the quantity of lines; $L_{m}(\gamma)$ means the length of the $m$ th attainable circle. $L$ means the length of the whole attitude circle. $D=1$ also means that, at this point, any attitude can be reached with the joints in their rotational extent. The unit of point that can make $D=1$ is also called Omnidirectional space.

3.3. Mission Attitude Constraints. Mission attitude constraints define manipulator attitude mathematical description based on a specific mission. The constraints explain all attitudes of an end-effector required for completing mission. The point meeting mission attitude constraints is called Omnidirectional point of special task, which consists of mission attitude space. It is obvious that different missions have different mission attitude spaces for the same manipulator.

The constraints are described with subspace for one continuous sphere surface, such as $S_{m}$, as shown in Figure 6. In order to establish the constraint equations, we set up the mapping between the sphere surface and plane, as shown in Figure 7.

Assuming the corresponding $\alpha$ range $[a, b]$, we equally divide the range into $k-1$ parts. The left point is $M_{1}$, the right point is $M_{k}$, and the number of points is $j(j=2 k-2)$. $M_{i}$ is expressed with $\left(\alpha_{i}, \beta_{i}\right)$; then constraint equation of $C_{i}$ is shown as follows.

When $i=1$,

$C_{1}$

$$
=\left\{\begin{array}{l}
\left(\beta_{2}-\beta_{1}\right) \alpha-\left(\alpha_{2}-\alpha_{1}\right) \beta+\beta_{1} \alpha_{2}-\beta_{2} \alpha_{1} \leq 0 \\
\left(\beta_{n}-\beta_{1}\right) \alpha-\left(\alpha_{n}-\alpha_{1}\right) \beta+\beta_{1} \alpha_{n}-\beta_{n} \alpha_{1} \geq 0 \\
\alpha_{1} \leq \alpha<\alpha_{2}
\end{array}\right.
$$

when $2 \leq i \leq k-1$,

$$
C_{i}=\left\{\begin{array}{l}
\left(\beta_{i+1}-\beta_{i}\right) \alpha-\left(\alpha_{i+1}-\alpha_{i}\right) \beta+\beta_{i} \alpha_{i+1}-\beta_{i+1} \alpha_{i} \leq 0 \\
\left(\beta_{2 k-i-1}-\beta_{2 k-i}\right) \alpha-\left(\alpha_{2 k-i-1}-\alpha_{2 k-i}\right) \beta+\beta_{2 k-i} \alpha_{2 k-i-1}-\beta_{2 k-i-1} \alpha_{2 k-i} \geq 0 \\
\alpha_{i} \leq \alpha<\alpha_{i+1} ;
\end{array}\right.
$$




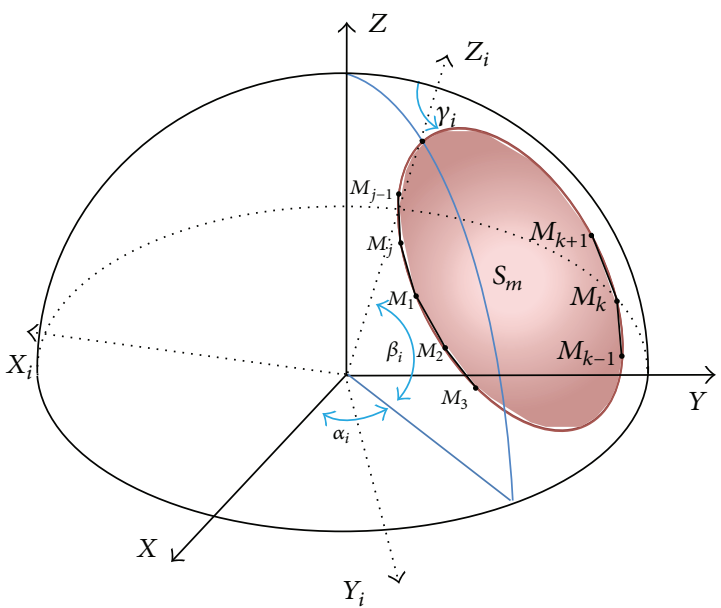

FIGURE 6: Task attitude constraints on sphere surface.

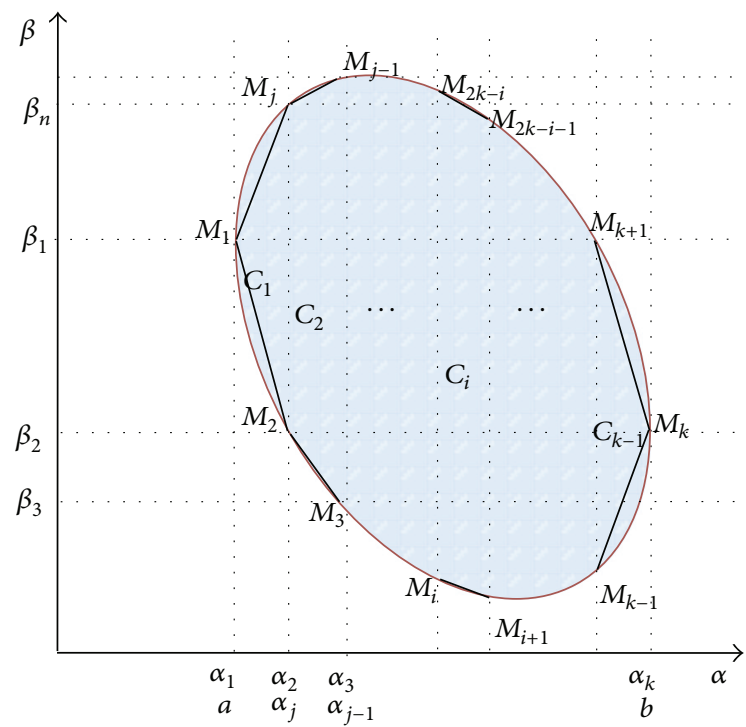

Figure 7: Task attitude constraints on plane.

then constraint equation of $S_{m}$ is

$$
C S_{m}=\sum_{i=1}^{k-1} C_{i}
$$

At last mission attitude constraints are expressed as

$$
C T=\sum_{m=1}^{n} \sum_{i=1}^{k-1} C_{i} .
$$

In this paper, we just discuss convex surface, and constraint equations of concave surface can be established with the same way.

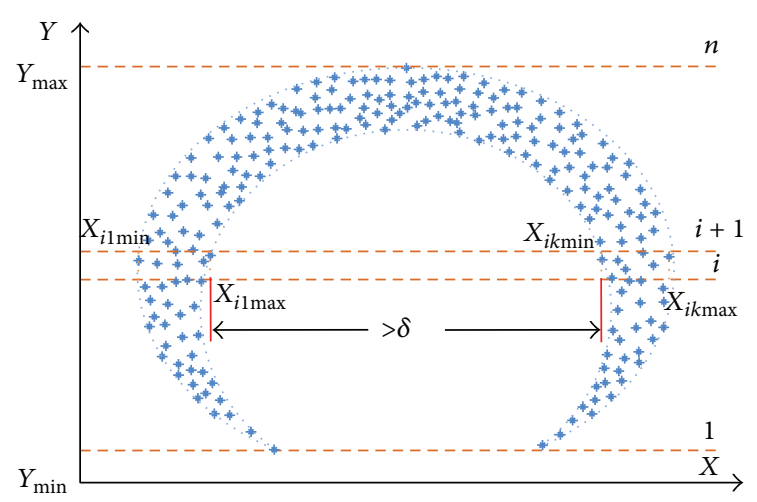

FIgUre 8: One slice of points.

For the 2D manipulator, we directly get the constraint equation (15) as follows, referring to Figure 5:

$$
C T=\sum_{m=1}^{n} \sum_{i=1}^{k} \gamma_{i} .
$$

According to special mission, we get mission attitude space, following these steps:

(1) Establish constraint equations to get CT in (14), based on special mission.

(2) Judge attitude of point $P_{i}$ which is included in attainable space meeting the entire $C T$.

(3) If it meets (2), $P_{i}$ is Omnidirectional point of special task; otherwise it is not. Then, we change $i$ to $i+1$ and repeat steps (1)-(3).

(4) All Omnidirectional points of special task consist of mission attitude space.

3.4. Volume of Point's Space. The volume of attainable space, mission attitude space, and Omnidirectional space all can reflect the manipulator performance and change with the manipulator parameters. Because they all calculate point's space, the same numerical analysis method is used.

It is complex to calculate the volume of $3 \mathrm{D}$ points, so we should deal with date by reducing dimension. The twodimensional point's distributions of each layer of the space are obtained by equally classifying these points into a series of slices along $Z$ axis, as shown in Figure 8 . We assume that height of each slice is $\xi=\left(Z_{\max }-Z_{\min }\right) / m$, where $Z_{\max }$ and $Z_{\text {min }}$ are maximum and minimum of all points, respectively, and $m$ means quantity of slices. Now, the three-dimensional volume problem turns into two-dimensional area, so we only need to calculate the size of the area of plane points.

For calculating point's area, we can use the method of curve fitting and curve integral; in addition, Fuchs algorithm that calculates the area is also feasible through triangulation. In this paper, we apply rectangle method, as shown in Figure 8 . The area is equally divided, and the width of each row is $\eta=\left(Y_{\max }-Y_{\min }\right) / n$, where $Y_{\max }$ and $Y_{\min }$ are maximum 
and minimum of points, respectively, and $n$ means quantity of rows.

According to the value of $X$, we arrange points in the $i$ th row, getting the series $X_{i 1}, X_{i 2}, \ldots, X_{i i}, X_{i i+1}, \ldots, X_{i w}$. Then we achieve distance series of adjacent points, $\delta_{i 1}$, $\delta_{i 2}, \ldots, \delta_{i i}, \delta_{i i+1}, \ldots, \delta_{i w-1}$.

We define a constant $\delta$ for judging holes phenomenon. If $\delta_{i i}>\delta$, it means there is a hole between $X_{i i}$ and $X_{i i+1}$. Holes make the series into $k$ segments, and $X_{i i \min }$ and $X_{i i \max }$ are maximum and minimum of $i$ th segment, as shown in Figure 8. When there is only one point in the row or each $\delta_{i i}>\delta$, we set the size of row area to zero.

The size of each row area is

$$
A_{z i}=\eta \sum_{j=1}^{k-1}\left[\left(X_{z i j \max }-X_{z i j \min }\right)\right] .
$$

The size of slice is

$$
\begin{aligned}
A_{z} & =A_{z 1}+A_{z 2}+\cdots+A_{z n} \\
& =\eta \sum_{i=1}^{n-1} \sum_{j=1}^{k-1}\left[\left(X_{z i j \max }-X_{z i j \min }\right)\right] .
\end{aligned}
$$

Then, the volume of point's space is

$$
V=\xi \sum_{z=1}^{m-1} A_{z}=\xi \eta \sum_{z=1}^{m-1} \sum_{i=1}^{n-1} \sum_{j=1}^{k-1}\left[\left(X_{z i j \max }-X_{z i j \min }\right)\right] \text {. }
$$

3.5. Manipulator Omnidirectional Coefficient. In the Omnidirectional space, the Omnidirectional Coefficient is 1 at all points. At the point in the domain between the attainable space and the Omnidirectional space, the Omnidirectional Coefficient is 0 to 1 . If we disperse the attainable space into $N$ points and assume POC as $D_{i}$ for $P_{i}$ correspondingly, the Manipulator Omnidirectional Coefficient can be expressed as follows:

$$
D A=\frac{\sum_{i=1}^{n} D_{i}}{N}
$$

The bigger $D A$ means the better of Omnidirectional characteristics of the manipulator; $D A=1$ means the attainable and the Omnidirectional space are the same.

We also express Manipulator Omnidirectional Coefficient based on special mission as follows:

$$
\mathrm{TDA}=\frac{\sum_{i=1}^{n} C D_{i}}{N}
$$

where $C D_{i}$ means Omnidirectional Coefficient of special mission. If the point is Omnidirectional point of special mission, $C D_{i}=1$; otherwise $C D_{i}=0$.

\section{Experiment}

Considering 3D manipulator and 2D manipulator, we can analyze them according to the method used above. They use the same analysis process and method, but also there
TABLE 1: DH parameters of manipulator.

\begin{tabular}{cccccccc}
\hline$i$ & $\theta$ & $\alpha$ & $d$ & $a$ & Initial limit & Final limit & Joint's type \\
\hline 1 & $*$ & $90^{\circ}$ & $L_{1}$ & 0 & $-120^{\circ}$ & $120^{\circ}$ & $\mathrm{R}$ \\
2 & $*$ & 0 & 0 & $L_{2}$ & $-80^{\circ}$ & $100^{\circ}$ & $\mathrm{R}$ \\
3 & $*$ & $-90^{\circ}$ & 0 & 0 & $-170^{\circ}$ & $10^{\circ}$ & $\mathrm{R}$ \\
4 & $*$ & $90^{\circ}$ & $L_{3}$ & 0 & $-180^{\circ}$ & $180^{\circ}$ & $\mathrm{R}$ \\
5 & $*$ & $-90^{\circ}$ & 0 & 0 & $-170^{\circ}$ & $10^{\circ}$ & $\mathrm{R}$ \\
6 & $*$ & 0 & 0 & 0 & $-180^{\circ}$ & $180^{\circ}$ & $\mathrm{R}$ \\
\hline
\end{tabular}

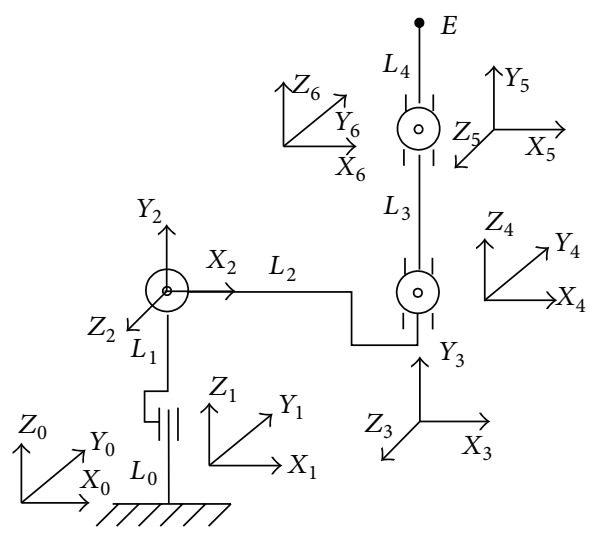

FIGURE 9: 3D manipulator structure.

are differences under the conditions that 3D manipulator performs in three-dimensional space and $2 \mathrm{D}$ manipulator performs in two-dimensional space. We could think that $2 \mathrm{D}$ manipulator is simplified from 3D manipulator.

4.1. 3D Manipulator. In this subsection, we will show the structure and explain some results of 3D manipulator.

4.1.1. System Description. The 3D manipulator structure is shown in Figure 9 and described with DH parameters illustrated in Table 1.

Where $L_{1}=0.08 \mathrm{~m}, L_{2}=0.34 \mathrm{~m}$, and $L_{3}=0.26 \mathrm{~m}$, we defined that $L_{0}=0.13 \mathrm{~m}$ is the distance between the origin of world coordinate system and first joint; $L_{4}=0.17 \mathrm{~m}$ means the distance between $n$th joint and end-effector.

4.1.2. Omnidirectional Analysis. The coordinate of initial position of the manipulator end-effector is $P=\left[\begin{array}{lll}0.34 & 0 & 0.64\end{array}\right]$, while the rotational angle is $q=[0,0,0,0,0,0]$. Disperse attitude sphere, and then represent the possible key as " $*$ ", showed in Figure 10. From (9), we can get the POC of the initial point valued 0.3946 .

According to the method used in solving POC, disperse the attainable space, and then get the Manipulator Omnidirectional Coefficient. Figure 11 shows the attainable space and the Omnidirectional space, where red “*” means Omnidirectional point and blue “*” represents the others. Analyzing it with the method used in (19) and in Section 3.4, we get that the Manipulator Omnidirectional Coefficient is 


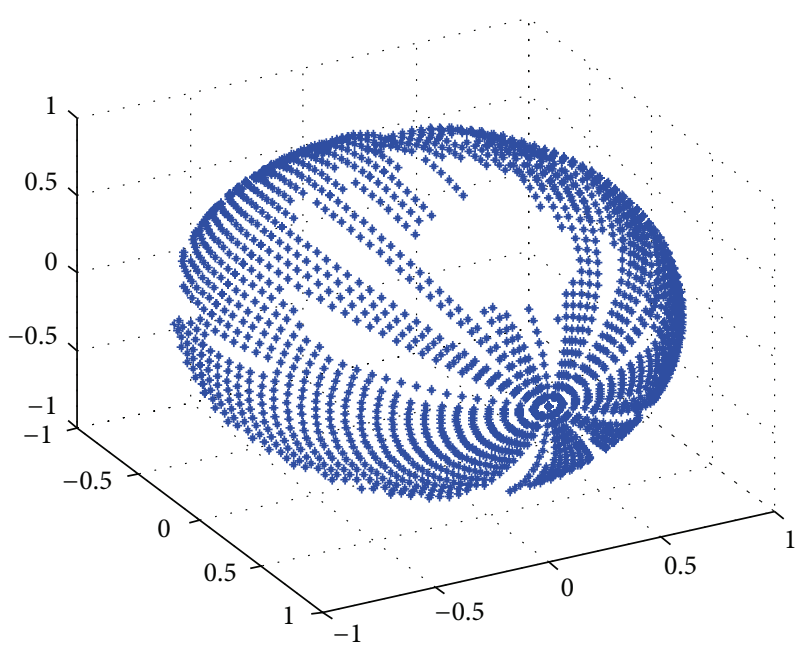

FIGURE 10: Reached attitude of initial position.

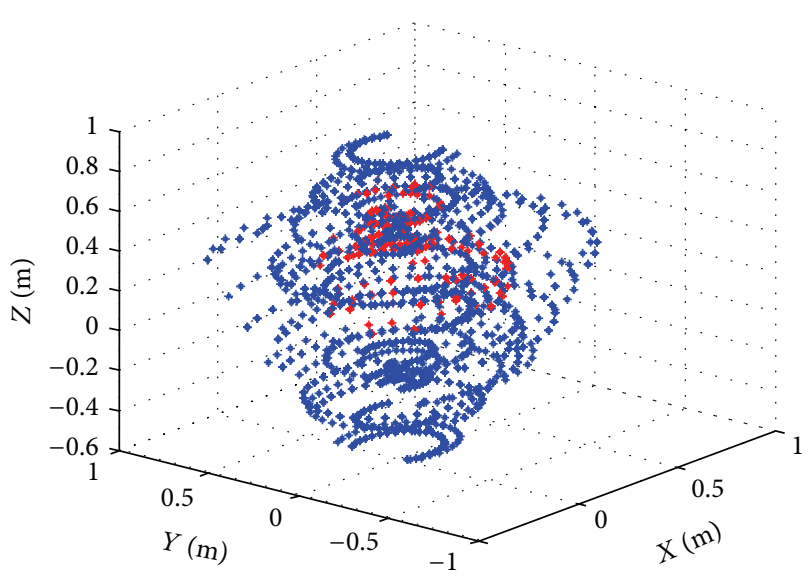

FIgURE 11: Attainable space and Omnidirectional space.

0.21 , the volume of attainable space is $0.243 \mathrm{~m}^{3}$, and the volume of Omnidirectional space is $0.008 \mathrm{~m}^{3}$.

The definition of the loading position is based on the analysis of Omnidirectional Coefficient. When the test platform is flat, the installed loading operation point must set in one flat. Assume the flat is $Z=0.21 \mathrm{~m}$; then, the POC on the flat could be represented as in Figure 12. The figure shows that the deeper the color is, the bigger the coefficient is. It is more complicated to operate loading, so we can set its operation point into the deeper colorful domain in order to complete the work. Considering the multiloading installation, we need an analysis about the complexity of the loading operation on one hand; and on the other hand, an Omnidirectional analysis about different loading flat is also essential, so that we can get the best distribution.

4.1.3. Omnidirectional Analysis Based on Task. The above subsections have discussed attitude constraint equations,

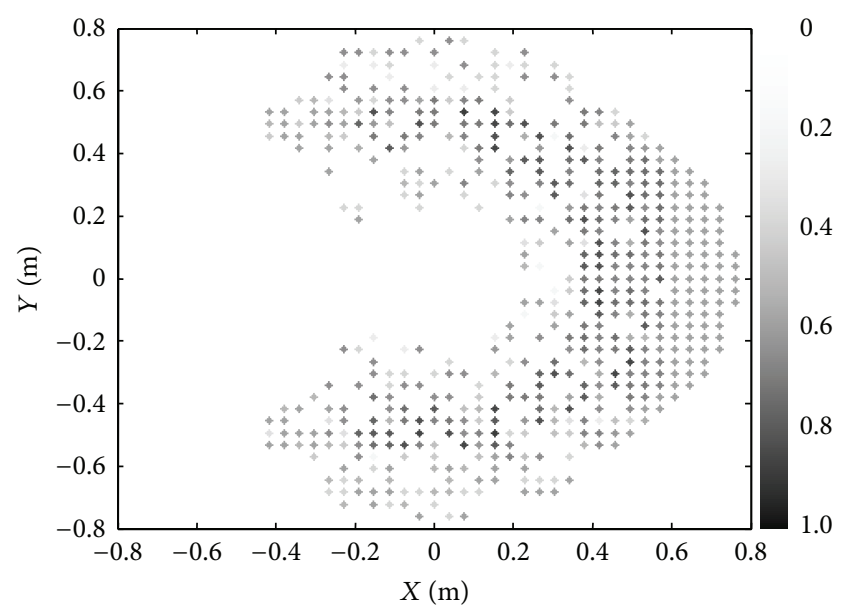

Figure 12: Omnidirectional Coefficient of plane $Z=0.21 \mathrm{~m}$.

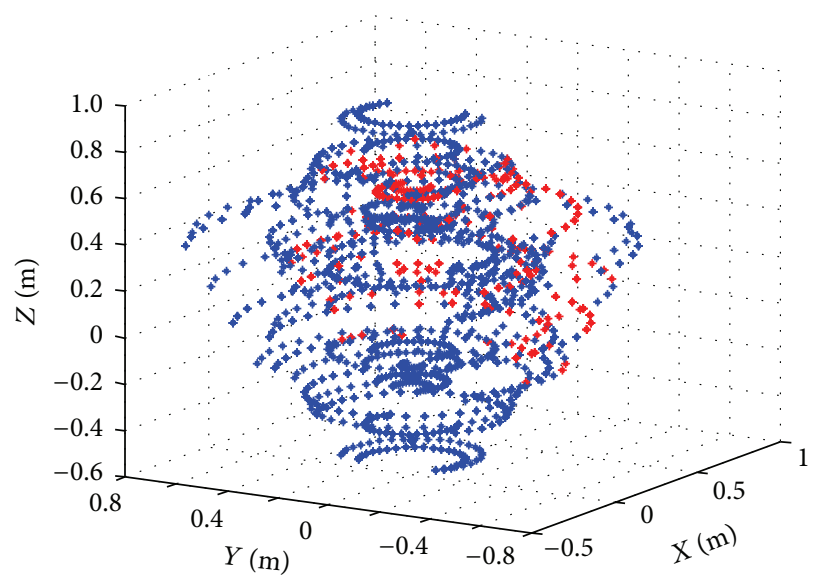

FIGURE 13: Attainable space and mission attitude space.

Omnidirectional Coefficient of special task, and mission attitude space. In this subsection, we assume attitude constraint equations as

$$
C T=\left\{\begin{array}{l}
\alpha \in\left[0, \frac{\pi}{2}\right] \\
\beta \in\left[0, \frac{\pi}{2}\right] .
\end{array}\right.
$$

It expresses one-eighth of attitude sphere. Disperse the attainable space and the Omnidirectional point is represented as red "*", while the others are in blue "*", as shown in Figure 13. Besides, mission attitude space is $0.024 \mathrm{~m}^{3}$.

\subsubsection{Relationship between Omnidirectional Characteristics} and Link Parameters. Link parameters include lengths of links, type of joints, and joint angle ranges, which fully impact Omnidirectional characteristics. In this subsection, we force the relationship about link lengths, joints angles, volume of attainable space, and Manipulator Omnidirectional Coefficient. 


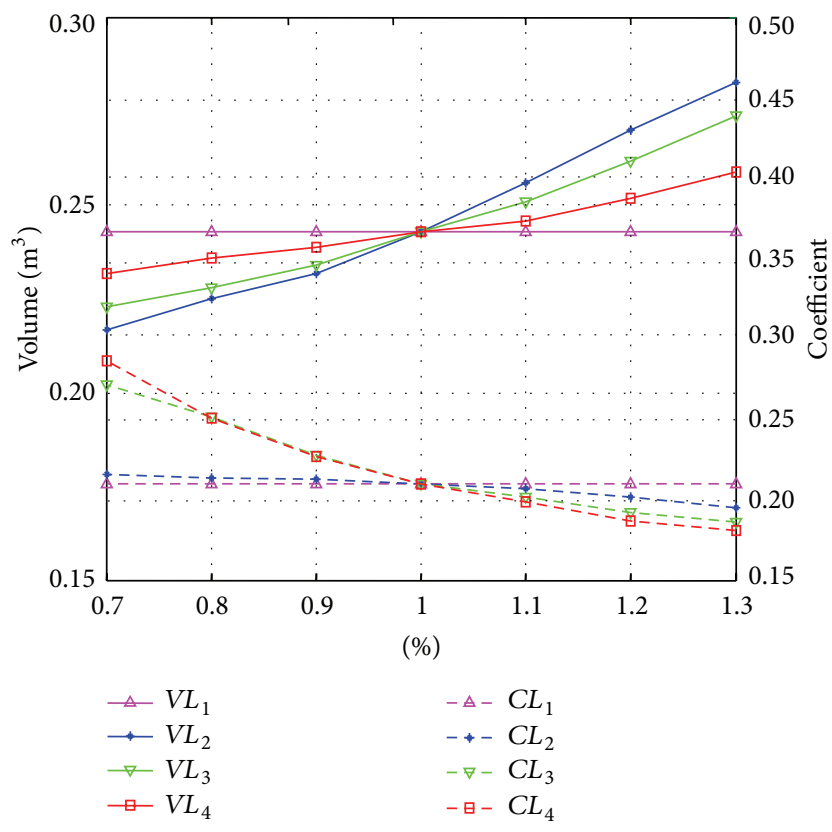

FIGURE 14: Relationship about link lengths, attainable space, and Manipulator Omnidirectional Coefficient.

In order to unifying, links lengths and joints angles are described in scale. For example, if the range of joint angle is $[a, b]$, percentage change will be expressed as

$$
\begin{array}{cc}
{[P a, P b],} & \text { if } a \leq 0 \leq b, \\
{[P a, b],} & \text { if } a \leq b \leq 0, \\
{[a, P b],} & \text { if } 0 \leq a \leq b,
\end{array}
$$

where $P$ means percentage, set as $0.7,0.8,0.9,1.0,1.1,1.2$, and 1.3 in this paper. Figure 14 represents relationship about link lengths, volume of attainable space, and Manipulator Omnidirectional Coefficient. Figure 15 shows relationship about joints angles, volume of attainable space, and Manipulator Omnidirectional Coefficient.

In Figure 14, $V L_{i}$ and $C L_{i}$ represent the curve of volume and percentage of $L_{i}(i=1,2,3,4)$ and the curve of coefficient and $L_{i}$, respectively. In Figure 15, $V L_{i}$ and $C L_{i}$ represent the curve of volume and percentage of joint angle $\theta_{i}(i=$ $1,2, \ldots, 6)$ and the curve of coefficient and $\theta_{i}$, respectively.

According to Figures 14 and 15, we would change our parameter for design requirements.

4.2. $2 D$ Manipulator. In this section, we will show the structure and analyze 3DOF 2D Manipulator Omnidirectional characteristics.

4.2.1. System Description. The 2D manipulator structure is shown in Figure 16 and described with DH parameters illustrated in Table 2.

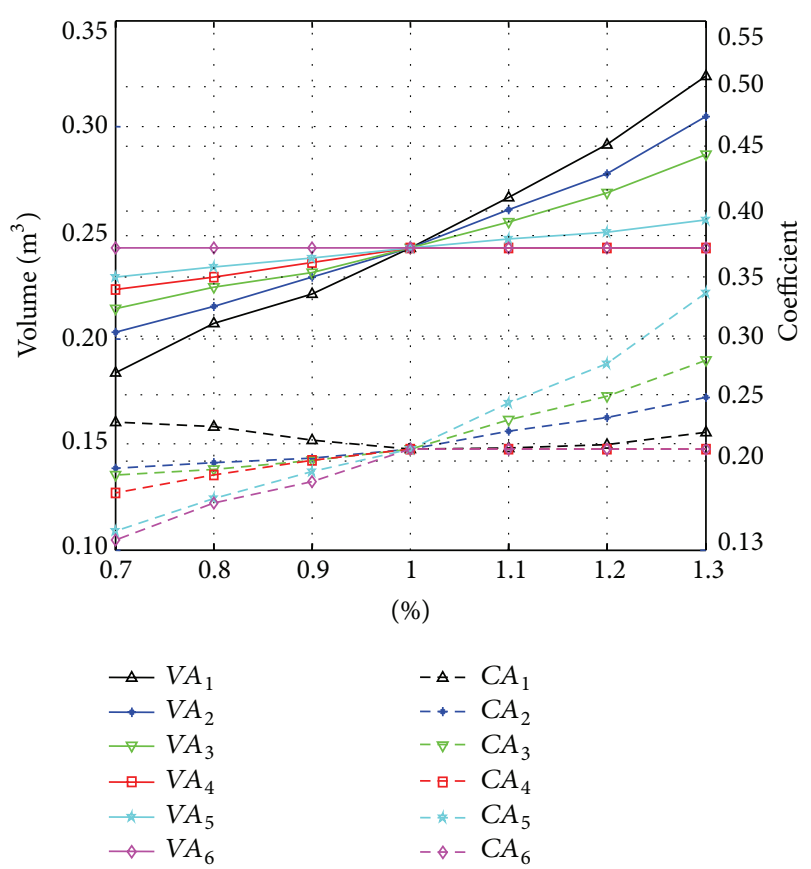

FIGURE 15: Relationship about joints angles, attainable space, and Manipulator Omnidirectional Coefficient.

TABLE 2: DH parameters of planar manipulator.

\begin{tabular}{cccccccc}
\hline$i$ & $\theta$ & $\alpha$ & $d$ & $a$ & Initial limit & Final limit & Joint's type \\
\hline 1 & $*$ & 0 & $L_{1}$ & 0 & $-120^{\circ}$ & $120^{\circ}$ & $\mathrm{R}$ \\
2 & $*$ & 0 & $L_{2}$ & 0 & $-160^{\circ}$ & $160^{\circ}$ & $\mathrm{R}$ \\
3 & $*$ & 0 & 0 & 0 & $-170^{\circ}$ & $170^{\circ}$ & $\mathrm{R}$ \\
\hline
\end{tabular}

In Table 2, $L_{1}=0.2 \mathrm{~m}$ and $L_{2}=0.16 \mathrm{~m}$. We defined that $L_{0}=0.21 \mathrm{~m}$ is the distance between the original point of the basic system and the first joint and $L_{3}=0.12 \mathrm{~m}$ is the distance between the third joint and the end-effector.

4.2.2. Omnidirectional Analysis. The coordinate of initial position of the manipulator end-effector is $P=[0.41,0]$. Disperse attitude of the manipulator at this point, and represent the possible key as blue “*”, showed in Figure 17. From the figure, we can get the Omnidirectional Coefficient of $P$ value 0.72 . For the $2 \mathrm{D}$ manipulator, both the attainable space and the Omnidirectional space are in one surface. Disperse the attainable space, and get the Omnidirectional Coefficient of all points; the point is represented as black "*”. Figure 18 shows that the deeper the color is, the bigger the Omnidirectional Coefficient is. We can know that the Omnidirectional Coefficient is 0.34 by (19).

4.2.3. Omnidirectional Analysis Based on Task. In this subsection, we assume that attitude constraint equation is $\gamma \in$ $[0 \pi / 2]$, which expresses one-fourth of attitude circle. The point is Omnidirectional attainable represented as red "*”, 


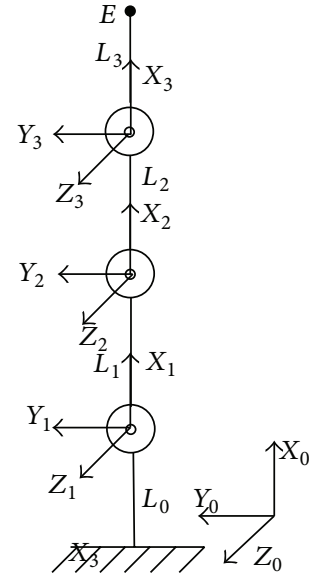

Figure 16: 3D manipulator structure.

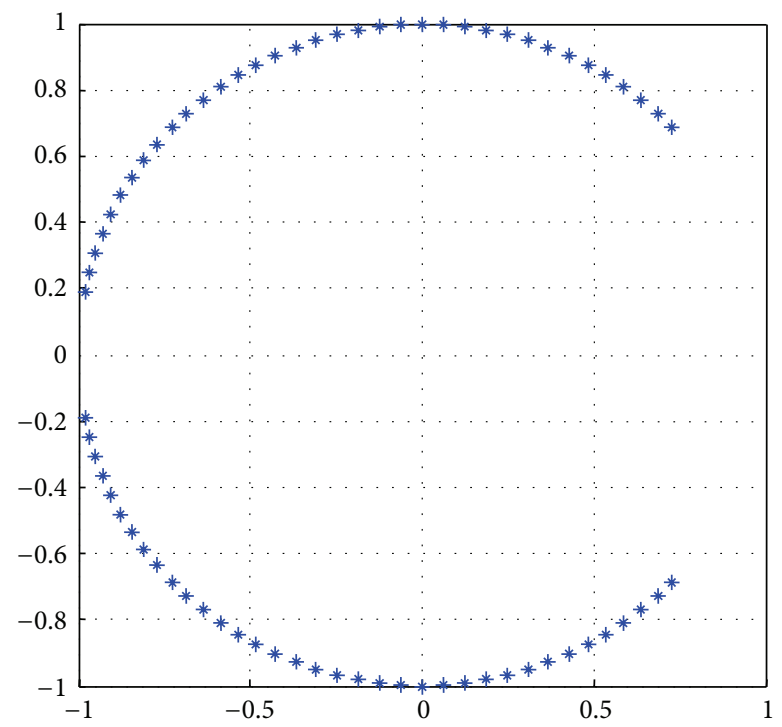

Figure 17: Reached attitude of $P$.

while the others are in black "*", as shown in Figure 19. Besides, mission attitude area is $0.056 \mathrm{~m}^{2}$.

4.2.4. Relationship between Omnidirectional Characteristics and Link Parameters. In this subsection we also discuss the relationship about link lengths, joints angles, volume of attainable space, and Manipulator Omnidirectional Coefficient, like in Section 4.1.4. Figure 20 represents relationship between link lengths, volume of attainable space, and Manipulator Omnidirectional Coefficient. Figure 21 shows relationship about joints angles, volume of attainable space, and Manipulator Omnidirectional Coefficient.

In Figures 20 and $21, A L_{i}$ and $A A_{i}$ mean area and percentage of $L_{i}(i=1,2,3)$ and the curve of area and $\theta_{i}(i=1,2,3)$, respectively, and others are the same as in Section 4.1.4.

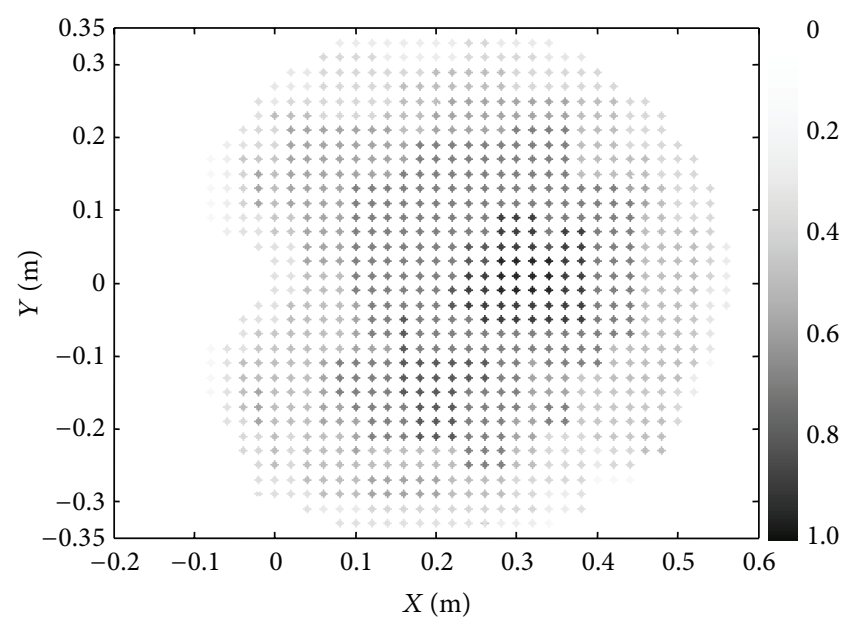

Figure 18: Omnidirectional Coefficient of plane.

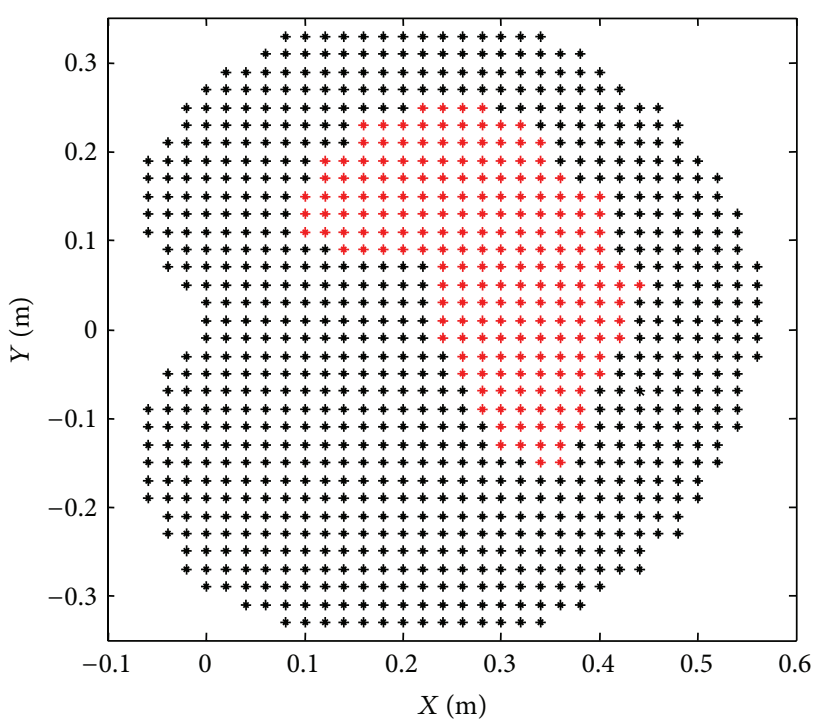

Figure 19: Attainable space and mission attitude area.

\section{Conclusion}

First, this paper proposes the POC with unit attitude sphere/circle to evaluate attitude function of the manipulator at one point; second, it represents the mapping method to establish mission attitude constraints function; third, it provides method for calculating volume of points space by reducing dimension; in addition, the Manipulator Omnidirectional Coefficient based on mission or not is proposed for evaluating manipulator performance. Under the condition of Omnidirectional characteristics analysis, it could be used in guiding the manipulator design and loading operation.

\section{Conflict of Interests}

The authors declare that there is no conflict of interests regarding the publication of this paper. 


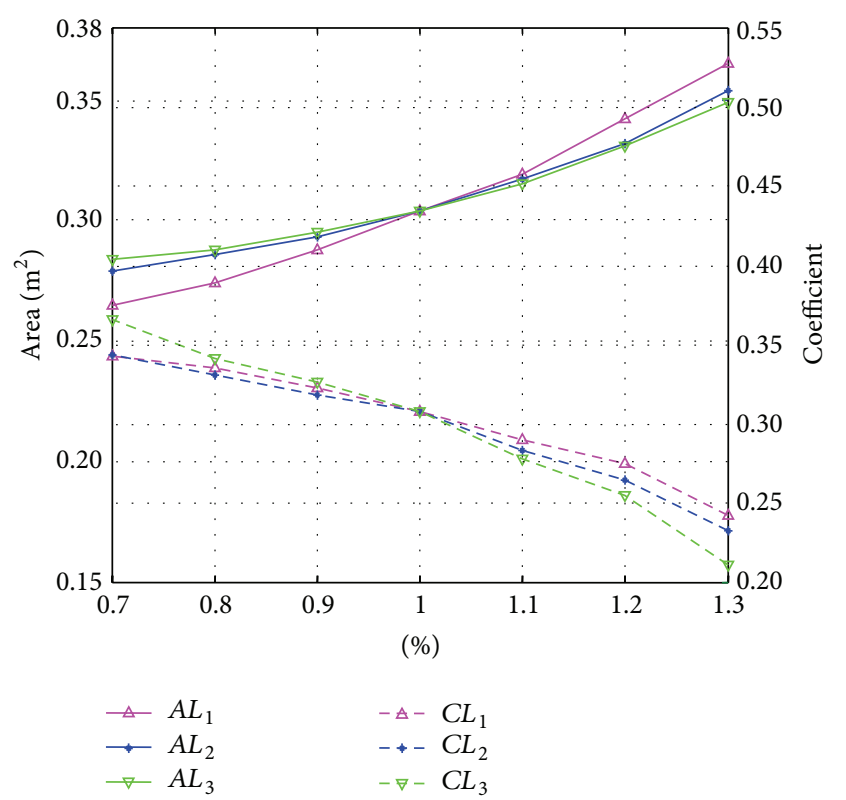

Figure 20: Relationship about link lengths, attainable space, and Manipulator Omnidirectional Coefficient.

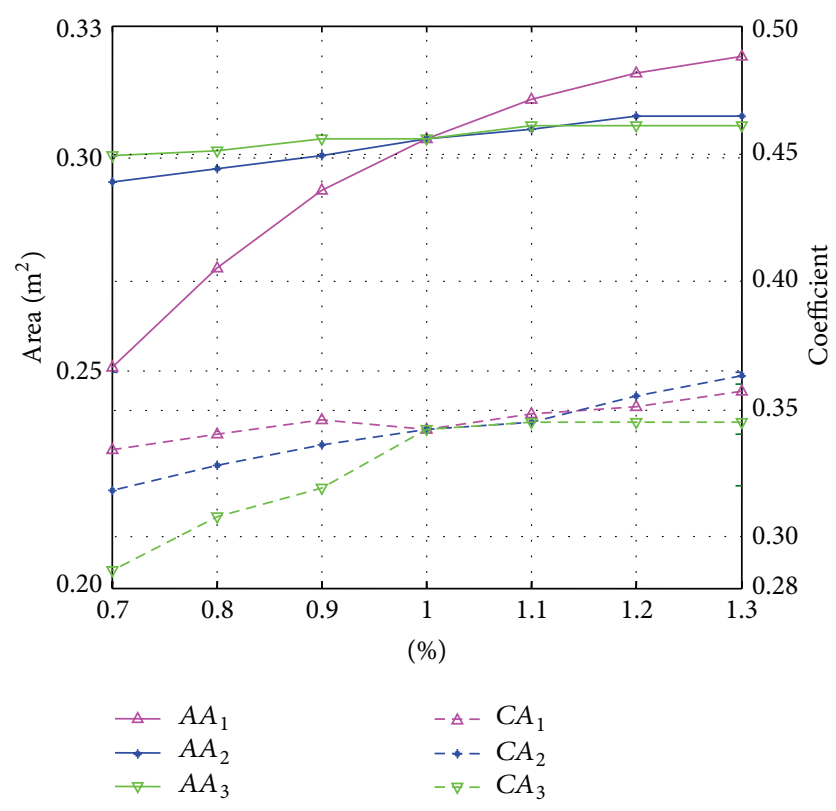

FIGURE 21: Relationship about joints angles, attainable area, and Manipulator Omnidirectional Coefficient.

\section{Acknowledgments}

This work is supported by National Postdoctoral Foundation of China under Grant 2013M541258, State Key Laboratory of Robotics (2015-Z08), and Doctoral Fund of Liaoning Province (20141152).

\section{References}

[1] K. C. Gupta and B. Roth, "Design considerations for manipulator workspace," Journal of Mechanical Design, vol. 104, no. 4, pp. 704-711, 1982.

[2] K. N. Umesh, "Dexterous mechanisms for robot locomotion," Mechanism and Machine Theory, vol. 33, no. 8, pp. 1153-1165, 1998.

[3] Z.-C. Lai and C.-H. Menq, "The dexterous workspace of simple manipulators," IEEE Journal of Robotics and Automation, vol. 4, no. 1, pp. 99-103, 1988.

[4] C. W. Qiu, L. M. Wang, and Y. W. Huang, "Multi-objective planning of continuous trajectory taskfor an omni-directional mobile dual-arm robot based on product arbitration," Robot, vol. 35, no. 2, pp. 178-185, 2013.

[5] K. Abdel-Malek, F. Adkins, H.-J. Yeh, and E. Haug, "On the determination of boundaries to manipulator workspaces," Robotics \& Computer-Integrated Manufacturing, vol. 13, no. 1, pp. 63-72, 1997.

[6] M. Mohammed, A. Y. Elkady, and T. Sobh, "A new algorithm for measuring and optimizing the manipulability index," Journal of Intelligent and Robotic Systems: Theory and Applications, vol. 59, no. 1, pp. 75-86, 2010.

[7] J. Yang, K. Abdel-Malek, and Y. Zhang, "On the workspace boundary determination of serial manipulators with nonunilateral constraints," Robotics and Computer-Integrated Manufacturing, vol. 24, no. 1, pp. 60-76, 2008.

[8] T. Yoshikawa, "Manipulability of robotic mechanisms," The International Journal of Robotics Research, vol. 4, no. 2, pp. 39, 1985.

[9] A. Kumar and K. J. Waldron, "The workspaces of a mechanical manipulator," Journal of Mechanical Design, vol. 103, no. 3, pp. 665-672, 1981.

[10] S. Kucuk and Z. Bingul, "Robot workspace optimization based on a novel local and global performance indices," in Proceedings of the IEEE International Symposium on Industrial Electronics (ISIE '05), pp. 1593-1598, Dubrovnik, Croatia, June 2005.

[11] D. Alciatore and C. Ng, "Determining manipulator workspace boundaries using the Monte Carlo method and least squares segmentation," ASME Robotics: Kinematics, Dynamics and Controls, vol. 72, pp. 141-146, 1994.

[12] Z. Gao, X. Lan, and Y. Bian, "Structural dimension optimization of robotic belt grinding system for grinding workpieces with complex shaped surfaces based on dexterity grinding space," Chinese Journal of Aeronautics, vol. 24, no. 3, pp. 346-354, 2011.

[13] K. Van Den Doel and D. K. Pai, "Performance measures for robot manipulators: a unified approach," The International Journal of Robotics Research, vol. 15, no. 1, pp. 92-111, 1996.

[14] C. M. Gosselin, "Dexterity indices for planar and spatial robotic manipulators," in Proceedings of the IEEE International Conference on Robotics and Automation, vol. 1, pp. 650-655, IEEE, Cincinnati, Ohio, USA, May 1990.

[15] A. J. Cebula and P. J. Zsombor-Murray, "Formulation of the workspace equation for wrist-partitioned spatial manipulators," Mechanism and Machine Theory, vol. 41, no. 7, pp. 778-789, 2006.

[16] C. Gosselin and J. Angeles, "Global performance index for the kinematic optimization of robotic manipulators," Journal of Mechanisms, Transmissions, and Automation in Design, vol. 113, no. 3, pp. 220-226, 1991. 
[17] X.-J. Liu, J. S. Wang, and F. Gao, "Workspace atlases for the design of spherical 3-DOF serial wrists," Journal of Intelligent and Robotic Systems, vol. 36, no. 4, pp. 389-405, 2003.

[18] J. K. Salisbury and J. J. Craig, "Articulated hands: force control and kinematic issues," The International Journal of Robotics Research, vol. 1, no. 1, pp. 4-17, 1982.

[19] C. Gosselin and J. Angeles, "The Optimum kinematic design of a spherical three-degree-of-freedom parallel manipulator," ASME Journal of Mechanism, Transmission and Automation in Design, vol. 111, no. 2, pp. 202-207, 1989.

[20] C. A. Klein and B. E. Blaho, "Dexterity measures for the design and control of kinematically redundant manipulators," The International Journal of Robotics Research, vol. 6, no. 2, pp. 7283, 1987.

[21] W. Z. Guo and F. Gao, "Solution space atlases, workspace characteristics charts and joint space maps for the design of planar serial manipulators," Mechanism and Machine Theory, vol. 45, no. 3, pp. 392-407, 2010.

[22] W. Z. Guo, F. Gao, and S. Mekid, "A new analysis ofworkspace performances and orientation capability for 3-dof planar manipulators," International Journal of Robotics and Automation, vol. 25, no. 2, pp. 89-101, 2010. 

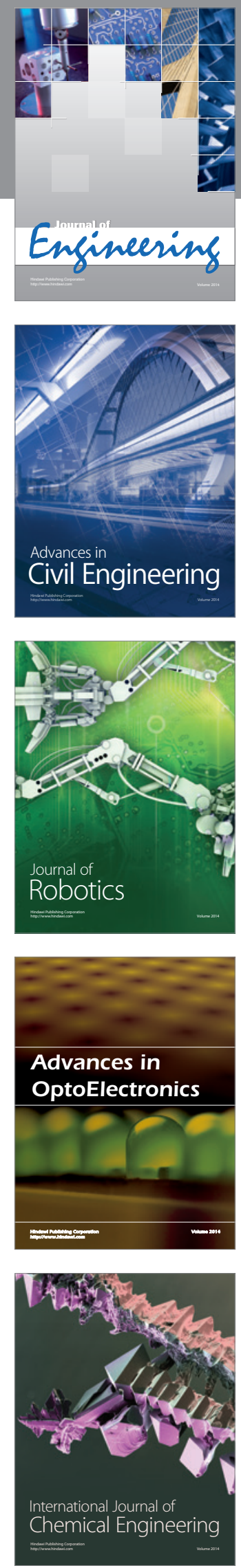

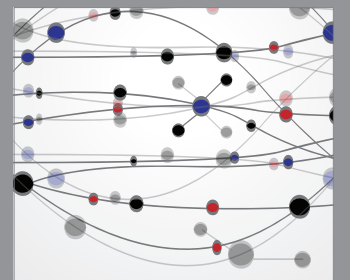

The Scientific World Journal
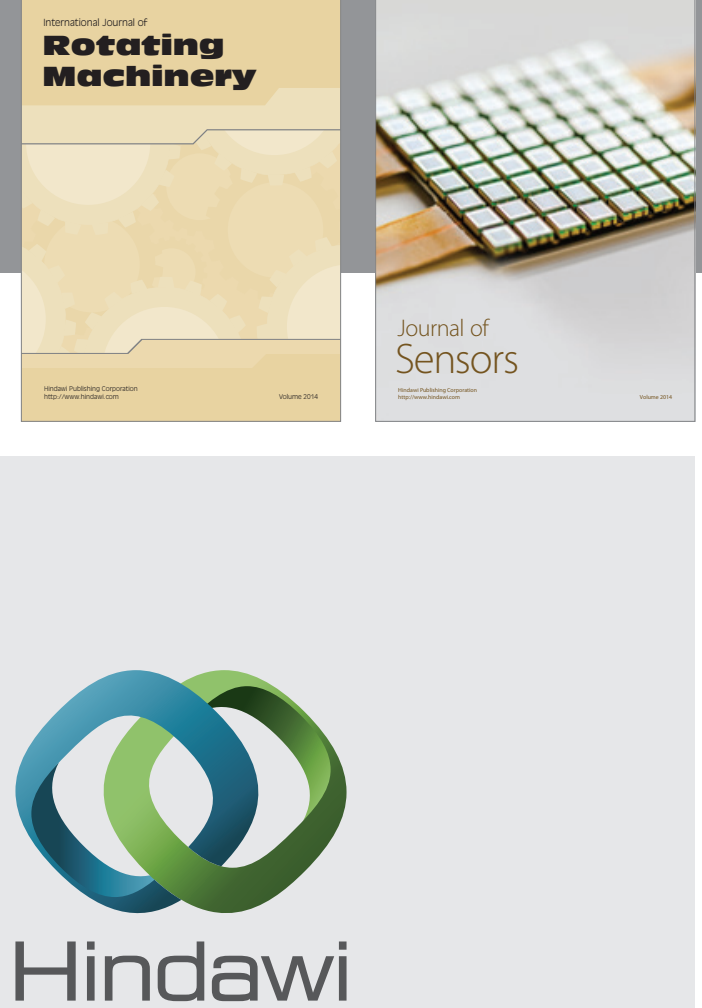

Submit your manuscripts at http://www.hindawi.com
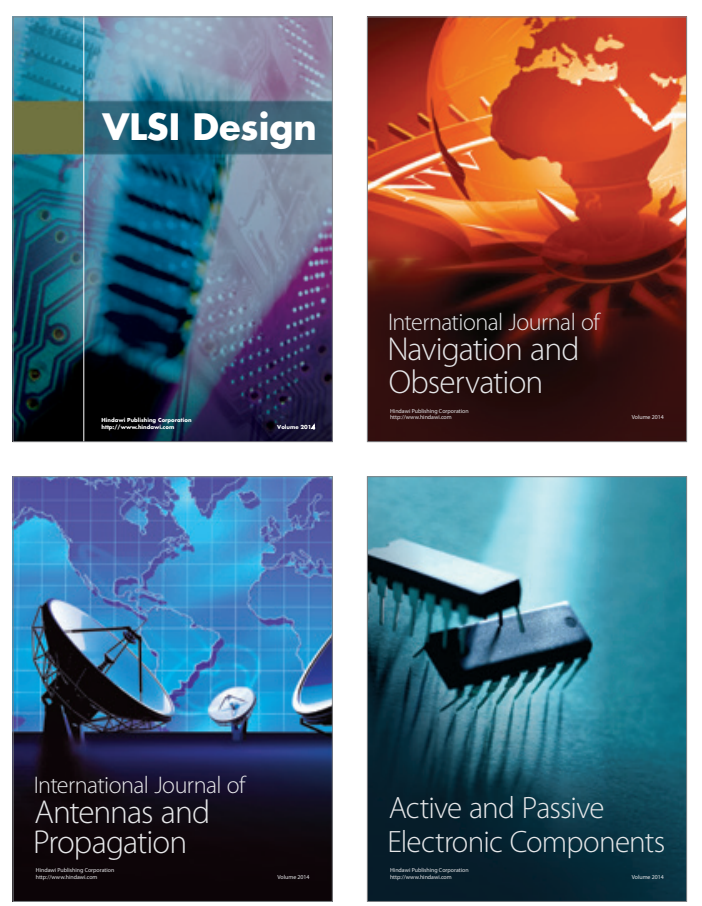
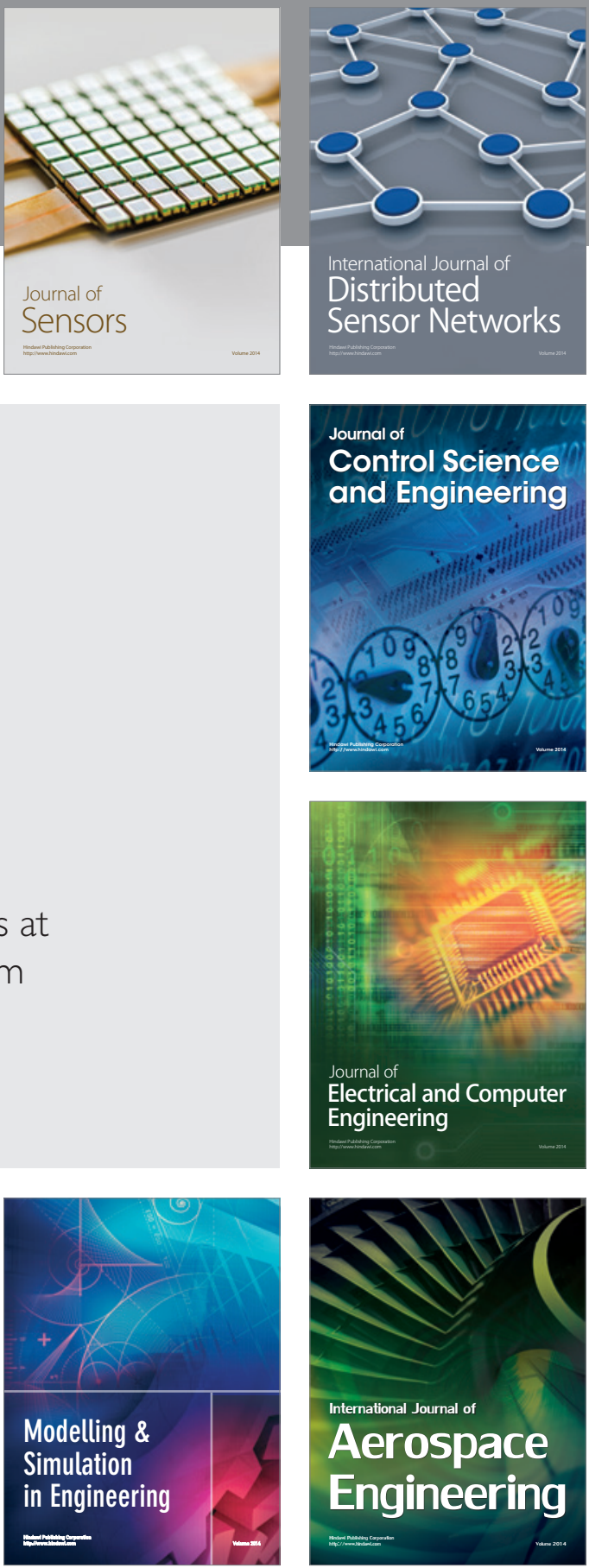

Journal of

Control Science

and Engineering
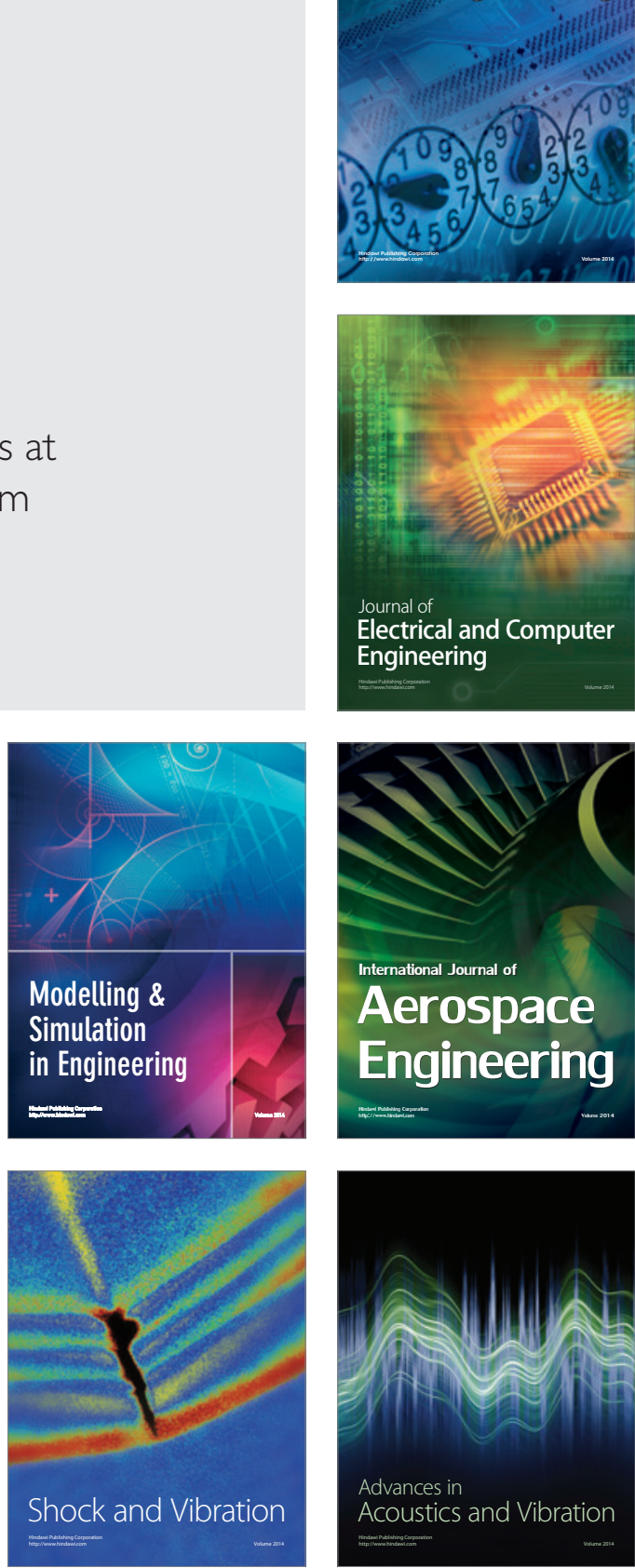